\title{
Comments on: Farkas' lemma: three decades of generalizations for mathematical optimization
}

\author{
Marco A. López
}

Published online: 1 February 2014

(C) Sociedad de Estadística e Investigación Operativa 2014

I am thankful to the Editor-In-Chief of Top, Prof. M. A. Goberna, for his kind invitation to write a comment to this invited article. My gratitude to him obeys two main reasons. The first one is that Farkas' Lemma (and related topics) is one of the main tools in my research along the years, and also a key stone in my teaching of optimization. The second one comes from my deep professional respect and recognition to these outstanding researchers, that I consider also my friends, after an extremely satisfactory collaboration along the years, whose fruitful outcomes were gathered in several joint papers.

I met Prof. Jeyakumar (together with Prof. Rubinov) for the first time during the International Conference on Nonlinear and Variational Inequalities, held in Hong Kong in 1998, although at this time I was already following his research trajectory through his publications. In fact, after one of my first papers, with E. Vercher, dealing with convex semi-infinite games (López and Vercher 1986), he published a nonconvex counterpart of our work (Jeyakumar 1988), but my definite interest in his research comes from the double fact that his topics are close to mines, and more important, because I like very much the kind of mathematics he does. I visited him in his university (New South Wales, Sydney) in 2009, after the 4th Australia-China Workshop on Optimization, held in Ballarat. During that short visit he told me that uncertainty and robustness in optimization attracted him as they have a real interest in applications, and we made plans for our second joint paper ([46], with G. Li and M. A. Goberna). That paper includes Theorem 2.4 in this survey, which is nothing else but a robust Farkas' lemma in the semi-infinite framework.

This comment refers to the invited paper available at doi:10.1007/s11750-014-0319-y.

M. A. López (ه)

Department of Statistics and Operations Research, University of Alicante, Alicante, Spain e-mail: marco.antonio@ua.es 
My professional relationship with Prof. Dinh started after a visit of M.A. Goberna to the University of South Wales. Both, Dinh and Goberna, collaborated with Jeyakumar and wrote a joint paper (Goberna et al. 2006). After that occasion, Dinh has been our frequent visitor in Alicante, and we established a strong link with him, leading to a permanent collaboration which has produced eight joint papers up to now (some of them with Profs. M. Volle, of the Université d'Avignon, and Prof. E. Ernst, from the Université de Marseille). Dinh became a member of our research team, granted by the Spanish Research System. In conclusion, I would like to say that writing this comment for Top is, simultaneously, a big challenge and a great pleasure.

In this paper the authors survey a broad family, a catalogue I would say, of generalizations of the celebrated Farkas's lemma. They range from systems of linear inequalities to a variety of nonlinear systems, and focus on the main applications of generalized Farkas' lemmas to continuous optimization problems. In my opinion, this survey constitutes a brilliant contribution to the field of mathematical optimization and, at the same time, a helpful tool for the interested researcher, who will find in it an exhaustive information on the topic. This is why I would like to congratulate both authors, and also to our journal Top, for the many expected benefits derived from the publication of this paper.

To the risk of an excessive simplification, I would say that in the process of deriving necessary optimality conditions for any optimization problem we can distinguish two fundamental steps. The first step is, in a huge number of models, the application of some version of Farkas' lemma. Under a simple set of assumptions, this first step gives rise to asymptotic optimality conditions via the so-called sequential forms of Farkas' lemma (see Section 7), where the existence of sequences (nets) satisfying certain limiting property is given as a characterization of the aimed property, in each case. But, if one looks for ordinary optimality conditions (of KKT-type), some (constraint) qualifications are needed, and this is the aim of the second step in the process. Some of these qualifications require the (weak*-)closedness of a certain convex set, as it happens in Theorems 2.1, 2.2, 2.4, 3.1, 4.1, 4.2, 4.8, 4.9, 5.1, and 6.1, and both authors are recognized specialists in handling these type of qualifications, since the publication of the papers [17] and [18] by R. Burachick and V. Jeyakumar. Other type of qualifications can be seen as (quasi-)interiority conditions and represent more or less sophisticated extensions (weakenings) of the simple Slater condition, so familiar for people working in convex optimization. An example of qualification of this kind is condition $(C Q)$ in Theorem 5.2, and they are commonly applied by other reputed specialists as R. Bot and his coauthors ([8] to [14]).

I would like to illustrate this two-step process by describing what happens in linear semi-infinite optimization (LSIP, in brief) where one wants to minimize the linear function $\langle c, x\rangle$ on a feasible set $F$ defined by means of infinitely many linear inequality constraints:

$$
\sigma:=\left\{\left\langle a_{t}, x\right\rangle \leq b_{t}, t \in T\right\}
$$

where $T$ is an arbitrary infinite set.

In the first step, we shall apply the corresponding LSIP-version of Farkas lemma (Goberna and López 1998) and we will get some asymptotic necessary optimality 
condition involving a sequence of sets of multipliers and its limiting behavior. These optimality conditions are not manageable at all, and we need to proceed to the second step. For this purpose, we introduce the following definition: The consistent $(F \neq \emptyset)$ system $\sigma$ is said to be a Farkas-Minkowski system (FM, in short) if every linear inequality $\langle a, x\rangle \leq b$ which is a consequence of $\sigma$ (i.e., it is satisfied by every feasible solution of $\sigma$ ) is a consequence of a finite subsystem of $\sigma$.

In Goberna and López (1998) [Theorem 5.3(i)] it is proved that the consistent system $\sigma$ is FM, if and only if

$$
\text { co cone }\left\{\left(a_{t}, b_{t}\right), t \in T ;\left(0_{n}, 1\right)\right\} \text { is closed in } \mathbb{R}^{n} \times \mathbb{R} \text {. }
$$

Let us observe that the FM property belongs to the class of closedness constraint qualifications since, applying the LSIP version of Farkas' lemma, it is straightforward to prove that any optimal point of the LSIP problem gives rise to a KKT-point, involving a finite set of positive multipliers (Goberna and López 1998, [Chapter 7]). Moreover, the FM property of $\sigma$ implies other properties of systems as strong CHIP at any point $\bar{x}$ of the feasible set $F$ (see, for instance, $\mathrm{Li}$ and $\mathrm{Ng}(2003,2005)$ ).

In Puente and Vera de Serio (1999), the consistent system $\sigma$ is said to be locally Farkas-Minkowski (LFM, in brief) at a point $\bar{x} \in F$ if every consequent linear inequality of $\sigma$ determining a supporting hyperplane to $F$ at $\bar{x}$ is also a consequence of a finite subsystem. Hence, FM implies LFM at any feasible point. It turns out that LFM is the weakest possible constraint qualification, and it is also of the closedness type since it is equivalent to the closedness of the so-called active cone at $\bar{x}$ (Goberna and López 1998, Chapter 5). Moreover, LFM is equivalent to strong CHIP.

The FM property is extended from linear to a consistent convex inequality system $\sigma:=\left\{f_{t}(x) \leq 0, t \in T\right\}$, which can be linearized using the Fenchel conjugates $f_{t}^{*}, t \in T$, or alternatively, using subdifferentials. In Li et al. (2000) and Li and $\mathrm{Ng}$ (2003), the LFM property for convex systems is called basic constraint qualification (BCQ).

Meanwhile, in the linear case, LFM and strong CHIP are equivalent, in the convex case, LMF implies strong CHIP. Conditions for the equivalence of both constraint qualifications are given in $\mathrm{Li}$ and $\mathrm{Ng}$ (2003) [Remark 2.2]. An exhaustive study of the relationships among the differently many CQ's in convex programming is carried out in Fang et al. (2010).

I would like to close this comment by thanking both the authors for their remarkable clarification of the subject they have offered, by means of this article, to the community of mathematical optimizers.

\section{References}

Fang DH, Li C, Ng KF (2010) Constraint qualifications for optimality conditions and total Lagrange dualities in convex programming. Nonlinear Anal 73:1143-1159

Goberna MA, Jeyakumar V, Dinh N (2006) Dual characterizations of set containments with strict convex inequalities. J Global Optim 34:33-54

Goberna MA, López MA (1998) Linear semi-infinite optimization. In: Wiley series in mathematical methods in practice, 2. Wiley, Chichester

Jeyakumar V (1988) Nonconvex infinite games. Optimization 19:289-296 
López MA, Vercher E (1986) Convex semi-infinite games. JOTA 50:289-312

Li C, Ng KF (2003) Constraint qualification, the strong CHIP and best approximation with convex constraints in Banach spaces. SIAM J Optim 14:584-607

Li C, Ng KF (2005) Strong CHIP for infinite system of closed convex sets in normed linear spaces. SIAM J Optim 16:311-340

Li W, Nahak C, Singer I (2000) Constraint qualifications for semi-infinite systems of convex inequalities. SIAM J Optim 11:31-52

Puente R, Vera de Serio VN (1999) Locally Farkas-Minkowski linear semi-infinite systems. TOP 7:103-121 